\title{
Investigation of neuropsychological mechanism of fabric smoothness sensation
}

DOI:

10.1007/s12221-019-8592-x

\section{Document Version}

Accepted author manuscript

Link to publication record in Manchester Research Explorer

\section{Citation for published version (APA):}

Liao, X., Li, Y., Hu, J., Li, Q., \& Wu, X. (2019). Investigation of neuropsychological mechanism of fabric smoothness sensation. Fibers and Polymers. https://doi.org/10.1007/s12221-019-8592-x

\section{Published in:}

Fibers and Polymers

\section{Citing this paper}

Please note that where the full-text provided on Manchester Research Explorer is the Author Accepted Manuscript or Proof version this may differ from the final Published version. If citing, it is advised that you check and use the publisher's definitive version.

\section{General rights}

Copyright and moral rights for the publications made accessible in the Research Explorer are retained by the authors and/or other copyright owners and it is a condition of accessing publications that users recognise and abide by the legal requirements associated with these rights.

\section{Takedown policy}

If you believe that this document breaches copyright please refer to the University of Manchester's Takedown Procedures [http://man.ac.uk/04Y6Bo] or contact uml.scholarlycommunications@manchester.ac.uk providing relevant details, so we can investigate your claim.

\section{OPEN ACCESS}




\section{Fibers and Polymers}

\section{Investigation of neuropsychological mechanism of fabric smoothness sensation --Manuscript Draft--}

Manuscript Number:

Full Title:

Article Type:

Corresponding Author:

\section{Corresponding Author Secondary} Information:

Corresponding Author's Institution:

Corresponding Author's Secondary Institution:

First Author:

First Author Secondary Information:

Order of Authors:

\begin{tabular}{l} 
Order of Authors: \\
\hline Order of Authors Secondary Information:
\end{tabular}

Funding Information:
FIPO-D-18-00592R1

Investigation of neuropsychological mechanism of fabric smoothness sensation

Regular articles

Yi Li

University of Manchester

UNITED KINGDOM

University of Manchester

Xiao Liao

Xiao Liao

Yi Li

Junyan $\mathrm{Hu}$

Quanhai Li

Xinxing Wu

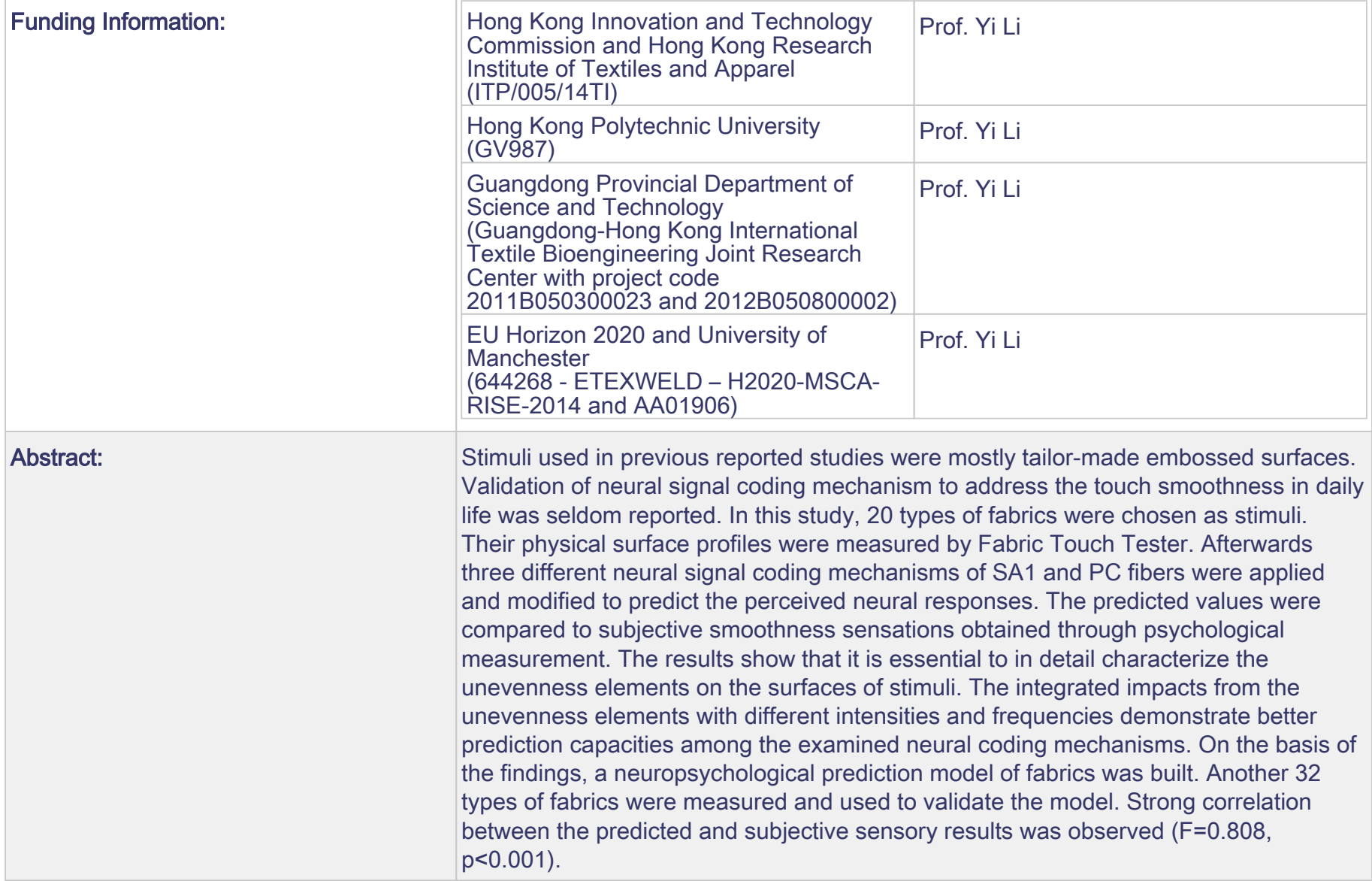


Reviewer \#1: Three hypotheses were evaluated from the physiological measurements and subjective tests. Since the smoothness perception should be due to a dynamic action of the finger, there is a limitation to correlate the physical and physiological measurements with the smoothness perception. The paper is not conclusive with regard to the preference of the hypotheses, but the paper is worth while being published. The authors should define at first the SA1 fiber and PC fiber because most of the readers are not familiar with the terms. Here the unevenness of the fabric was measured in one direction, but some of the fabrics used in the experiments have an anisotropic structure. Since the unevenness measurements is performed by a fine tip, it might different from the finger tip for the subjective text. However, the smoothness is concluded to be affected mainly by the surface properties of the fabric. I wonder whether the touching pressure and/or the finger moving direction would affect the sense of smoothness.

There are some typo-errors.

Reply:

Thank you very much for the valuable comments. Yes, the smoothness perception should be due to a dynamic action of the finger. Still, this study is important as it would further the study of touch perception into neuropsychological methods. Authors have planned next step studies into the dynamic perception of touch comfort through neuropsychological methods. The relevant contents were added into Conclusion Section.

Definitions of SA1 fiber and PC fiber were added into Introduction Section.

All sample fabrics were physically measured and subjectively evaluated in both warp and weft directions. More detailed description of the measuring principle, including pressure when measuring fabric surface properties, of Fabric Touch Test (FTT) and subjective evaluation were added into Material Section.

Typo-errors were corrected.

Reviewer \#2: 1. Introduction is relatively weak. More explanation for what SA1 and PC fiber means and how they are significant to human sensation is needed.

. Please address the principle of FTT and its functions even though it was introduced in a previous work.

3. Psychological measurement was not clearly stated in Materials. Please address in detail about its procedure, measurement scales, and questionnaire.

4. In Figure 8, authors argued that the relation between $\mathrm{W}$ and the smoothness sensation could be better fitted with logarithmic function. However readers cannot understand it because any other graphs such as $\mathrm{c}$ and d does not have R2 values or logarithmic function seen. Why don't you show R2 values of logarithmic functions or linear ones for graph $\mathrm{c}$ and $\mathrm{d}$ to identify which element would be more closely related to smoothness sensation by human?

5. The 20 fabrics in Table 1 are same to those used in reference [23]. Please cite reference [23] when you mention the fabrics firstly and clarify if the subjective sensation data were identical to those in reference [23] or not. Additionally describe the difference of this study from the reference [23] in "Introduction".

Reply:

1. Definitions of SA1 fiber and PC fiber were added into Introduction Section.

2. More detailed descriptions of the measuring principle of Fabric Touch Test (FTT) were added into Material Section.

3. More detailed descriptions of subjective measurement were added into Material Section.

4. In table 5, four defined indices I, W, P1, and P2 were examined and compared through correlation studies. It is found that $W$ has the highest coefficient value. Therefore, in Figure 8, only the relation between $W$ and psychological value was further investigated with linear and logarithmic function. Relevant contents were revised to make it clearer.

5. Citation was added. The subjective sensation data is not the same. More subjective experiment has been conducted. The difference between this study and previous study 
[23] (now [13]) was added into Introduction Section.

Powered by Editorial Manager ${ }^{\circledR}$ and ProduXion Manager ${ }^{\circledR}$ from Aries Systems Corporation 
Reviewer \#1: Three hypotheses were evaluated from the physiological measurements and subjective tests. Since the smoothness perception should be due to a dynamic action of the finger, there is a limitation to correlate the physical and physiological measurements with the smoothness perception. The paper is not conclusive with regard to the preference of the hypotheses, but the paper is worth while being published. The authors should define at first the SA1 fiber and PC fiber because most of the readers are not familiar with the terms. Here the unevenness of the fabric was measured in one direction, but some of the fabrics used in the experiments have an anisotropic structure. Since the unevenness measurements is performed by a fine tip, it might different from the finger tip for the subjective text. However, the smoothness is concluded to be affected mainly by the surface properties of the fabric. I wonder whether the touching pressure and/or the finger moving direction would affect the sense of smoothness.

There are some typo-errors.

\section{Reply:}

Thank you very much for the valuable comments. Yes, the smoothness perception should be due to a dynamic action of the finger. Still, this study is important as it would further the study of touch perception into neuropsychological methods. Authors have planned next step studies into the dynamic perception of touch comfort through neuropsychological methods. The relevant contents were added into Conclusion Section.

Definitions of SA1 fiber and PC fiber were added into Introduction Section.

All sample fabrics were physically measured and subjectively evaluated in both warp and weft directions. More detailed description of the measuring principle, including pressure when measuring fabric surface properties, of Fabric Touch Test (FTT) and subjective evaluation were added into Material Section.

Typo-errors were corrected. 
Reviewer \#2: 1. Introduction is relatively weak. More explanation for what SA1 and PC fiber means and how they are significant to human sensation is needed.

. Please address the principle of FTT and its functions even though it was introduced in a previous work.

3. Psychological measurement was not clearly stated in Materials. Please address in detail about its procedure, measurement scales, and questionnaire.

4. In Figure 8, authors argued that the relation between $W$ and the smoothness sensation could be better fitted with logarithmic function. However readers cannot understand it because any other graphs such as $\mathrm{c}$ and d does not have R2 values or logarithmic function seen. Why don't you show R2 values of logarithmic functions or linear ones for graph $\mathrm{c}$ and $\mathrm{d}$ to identify which element would be more closely related to smoothness sensation by human?

5. The 20 fabrics in Table 1 are same to those used in reference [23]. Please cite reference [23] when you mention the fabrics firstly and clarify if the subjective sensation data were identical to those in reference [23] or not. Additionally describe the difference of this study from the reference [23] in "Introduction".

\section{Reply:}

1. Definitions of SA1 fiber and PC fiber were added into Introduction Section.

2. More detailed descriptions of the measuring principle of Fabric Touch Test (FTT) were added into Material Section.

3. More detailed descriptions of subjective measurement were added into Material Section.

4. In table 5, four defined indices I, W, P1, and P2 were examined and compared through correlation studies. It is found that $W$ has the highest coefficient value. Therefore, in Figure 8 , only the relation between $\mathrm{W}$ and psychological value was further investigated with linear and logarithmic function. Relevant contents were revised to make it clearer.

5. Citation was added. The subjective sensation data is not the same. More subjective experiment has been conducted. The difference between this study and previous study [23] (now [13]) was added into Introduction Section. 
Title: Investigation of neuropsychological mechanism of fabric smoothness sensation

Authors: Xiao Liao ${ }^{1,2,3}$, Yi $\mathrm{Li}^{* 2,4}$, Junyan $\mathrm{Hu}^{2}$, Quanhai $\mathrm{Li}^{2}$, Xinxing $\mathrm{Wu}^{2}$

Affiliation:

${ }^{1}$ Southern University of Science and Technology, Shenzhen, China

${ }^{2}$ The Hong Kong Polytechnic University, Hung Hom, Hong Kong

${ }^{3}$ The Hong Kong Research Institute of Textiles and Apparel, Hong Kong

${ }^{4}$ School of Materials, The University of Manchester, UK

Corresponding author’s E-mail: henry.yili@manchester.ac.uk

Running Head: Neuropsychological mechanism of fabric smoothness sensation

Abstract: Stimuli used in previous reported studies were mostly tailor-made embossed surfaces. Validation of neural signal coding mechanism to address the touch smoothness in daily life was seldom reported. In this study, 20 types of fabrics were chosen as stimuli. Their physical surface profiles were measured by Fabric Touch Tester. Afterwards three different neural signal coding mechanisms of SA1 and PC fibers were applied and modified to predict the perceived neural responses. The predicted values were compared to subjective smoothness sensations obtained through psychological measurement. The results show that it is essential to in detail characterize the unevenness elements on the surfaces of stimuli. The integrated impacts from the unevenness elements with different intensities and frequencies demonstrate better prediction capacities among the examined neural coding mechanisms. On the basis of the findings, a neuropsychological prediction model of fabrics was built. Another 32 types of fabrics were measured and used to validate the model. Strong correlation between the predicted and subjective sensory results was observed $(\mathrm{F}=0.808, \mathrm{p}<0.001)$.

Keywords: Smoothness sensation; neuropsychological; touch comfort; coding; prediction model. 


\section{Introduction}

Human is able to discriminate smoothness sensation of objects with even very similar texture [1]. Researchers have conducted a large number of studies to discover the underlying mechanism of smoothness perception. Psychophysical studies usually conducted behavioral measurements to build relationship between stimuli's physical properties and subjects' perceptions $[2,3]$. Through statistical correlations studies, well-accepted conclusion is that the smoothness sensation was largely related to the surface friction force and geometrical unevenness.

Neuroscientists explored the neural signal coding mechanism of smoothness sensation. Once an environmental event is detected, the responsible human sensory system will be stimulated. Receptors translate physical external environments into electrical neural signals. Afferent fibers are the key organics for determining the response to a stimulus as well as the transmission speed of coded neural signals. Afferent fibers could be divided into two categories: those that are slowly adapting and rapidly adapting [4]. There are in general four different types of afferent fibers: The slow adapting afferents slowly adapting type I (SA1) and slowly adapting type II (SA2); and the rapidly adapting afferents: rapidly adapting (RA) and Pacinian afferent (PC) fibers.

As shown in Figure 1, studies were conducted to reveal the process of smoothness perception and to explore the mathematic relations between the initial physical stimulus and final psychological perception. During these studies, responses of afferent fibers were recorded from primates and stimuli were Braille-like embossed dot with pre-designed features [5-12]. These studies came to conclusions of mainly two types of smoothness sensation coding mechanisms: spatial variances of SA1 fiber or temporal variances of PC fiber. 
However, the proposed mechanisms have not yet been applied into predictions of smoothness sensations on nature surfaces. Physically heterogeneous surface on our daily contacted objects are much more complex than the stimuli used in aforementioned neurophysiological experiments [1].

In a previous study, a new measurement method of fabric touch properties, Fabric Touch Tester (FTT) was proposed. For the purpose of examining the performance of FTT, 20 types of fabrics were measured by both FTT and human subjects. Strong correlations between FTT-measured physical indices and subjective-evaluated touch sensation were reported [13]. In this previous work, only mathematic correlations between fabric physical properties and subjective sensations were investigated through regression method. Nevertheless, it is important to clear the underlying mechanisms of their connections. In this study, the relations between physical properties and subjective evaluation results were further analyzed trough neuropsychological method. This study concluded a preferred neuropsychological mechanism, which could explain the transduction process from external physical stimuli to psychological sensation.

Figure 1

Figure caption 1

\section{Hypothesis}

\section{Hypothesis 1}

The spatial coding of SA1 fibers was the first hypothesis of texture perception mechanism through a series of studies $[9-11,14,15]$. The effect of the unevenness height was investigated by Blake et al. [16]. They found that the height of the unevenness element has a 
major effect on SA1 impulse rates if the element diameter is less than $2.0 \mathrm{~mm}$. An experiment by Yoshioka et al. [12] confirmed that grooved and ridge widths have an effect on surface roughness perception. Grooved and ridge widths could also be summed up as element width (or center-to-center distance). An interpolated equation from these two studies is linearly expressed as:

$$
I=\mathrm{b}+K_{h} S_{h}+K_{w} S_{w} \quad \text { Equation } 1
$$

where $I$ denotes the predicted impulse rates of a single SA1 fiber, $S_{h}$ and $S_{w}$ are the height and width of the unevenness element, $\mathrm{K}_{h}(166.437)$ and $\mathrm{K}_{w}(-5.957)$ are the coefficients of the height and width of the stimulus, and $b$ is a constant (116.508).

\section{Hypothesis 2}

Besides coding mechanisms based on spatial variances of SA1 fibers, questions have been raised regarding the effects of other afferent fibers. A duplex theory of roughness perception was proposed $[17,18]$. This theory agreed on the spatial encoding mechanism of smoothness sensation, however, only for coarse texture. It was proposed that vibrotactile encoding would be more appropriate for fine texture. Later studies indicated that minor unevenness at micrometer scale would lead to skin vibrations $[19,20]$. Such skin vibration would stimulate another type of skin receptor, the PC fibers, and induce smoothness sensations.

Bensmaia and Hollins examined the relations between perceived smoothness and peak frequency and intensive information (power of the vibrations-weighted) of skin vibration. They concluded preference on the latter one [20]. There was an index of the PC vibration weighted power proposed by Makous et al. [21]. It was found that the relations between perceived roughness and the weighted power of PC fiber follows a logarithmic function [22]. The index of the weighted power $(W)$ of PC fiber vibration is calculated by: 


$$
\mathrm{W}=\sum_{i=1}^{200} A_{i}^{2} f_{i}^{2} h_{i} \quad \text { Equation } 2
$$

where $A_{i}$ refers to the stimuli amplitude at frequency $f_{i}, f_{i}$ was set with a constant interval, 2 in the study [20], and $H_{i}$ denotes the weight of the sensitivity of the PC fibers . $H_{i}$ is given by

$$
H_{i}=\frac{\min \left(T_{1}^{2} f_{1}^{2} \ldots T_{i}^{2} f_{i}^{2}\right)}{T_{i}^{2} f_{i}^{2}} \quad \text { Equation } 3
$$

where $T_{i}$ denotes the PC fiber threshold at frequency $f_{i}$.

The thresholds of the PC fibers used by Bensmaia and Hollins [20] were interpolated from the findings of Bolanowski et al. in 1988 [23] and linearly extrapolated until $1500 \mathrm{~Hz}$. It was also reported that human skin vibrations basically mirror the geometric properties on the stimuli surface [20]. Clearly, this coding method requires a detailed characterization of the surface geometric properties of the stimuli.

\section{Hypothesis 3}

In a recent study, three different quantitative methods, used to predict the neural impulses, were summarized and compared [24]. These three methods include the firing rate of individual afferents; mean firing rate of the entire population of active afferents; and number of active afferents. It was concluded that the logarithmic of the population firing rate has the best fit for psychological sensation. The mean firing evoked in a particular population was calculated in accordance with the stimulus amplitude and threshold. The thresholds were found to be linearly correlated to the stimulus frequency in a certain range (10 to $200 \mathrm{~Hz}$ for PC and SA1 fibers). The concluded formula of the mean population rate of SA1 and PC fibers is:

$$
P=\frac{1}{N} \sum_{i=1}^{N}\left[k_{i}\left(\log _{10} A_{i}-\log _{10} T_{i}\right)\right] \quad \text { Equation } 4
$$


where $k_{i}, A_{\mathrm{i}}$ and $T_{i}$ are the coefficient, stimulus intensity and fiber threshold respectively. $\mathrm{N}$ stands for the total number of stimuli calculated. The values of $k_{i}$ and $T_{i}$ were extrapolated as a linear function of the frequencies from 10 to $1000 \mathrm{~Hz}$ [24].

\section{Materials}

\section{Stimuli}

20 fabrics were selected as stimuli in this study as listed in the following Table 1, which is the same as in the previous study [13]. Fabric Touch Tester (FTT) was used to characterize the surface properties of the fabrics. FTT is a fabric touch properties measurement apparatus which has a special design to ascertain simultaneous measurement of four types of fabric properties related to touch sensations: thermal, compression, bending, and surface.

FTT uses laser sensor to detect the geometric profile of fabric surface. A needle-shape detector is used as contact module with the fabric surface. It would move up and down along with the fabric surface unevenness and its moving distance is recorded by the lase sensor. Such method simulates actual touch of fabric by humans: a slight normal pressure would be applied. Both warp and weft direction of fabric sample are measured by FTT. Their average is used to represent the overall surface roughness properties. A typical output curve is illustrated in following figure 2. X-axis of the figure represents measured distance of sample while Y-axis indicates geometric heights on its surface. Two indices named Surface Roughness Amplitude (SRA) and Surface Roughness Wavelength (SRW) are identified in FTT [13]. They represent the average amplitude and wavelength of the measured geometric patterns.

Table 1 
Figure 2

Figure caption 2

\section{Characterization of Stimuli}

The height and width of stimulus were comparatively easier to identify on the embossed surfaces. However for fabrics, a method should be designed to identify the height and width of the surface unevenness elements. FTT defined two indexes as SRA and SRW to describe the fabric surface properties. They simplified fabric surface profile as repeated patterns that similar to sinusoid, as shown in the following Figure 3. With this stimulus characterization method, the impulses rate of SA1 fiber could be predicted through Eq. 1.

Figure 3

Figure caption 3

The weaving nature of fabrics is interlacing of two groups of yarns. The surface unevenness elements on fabrics could be due to 1) the chemicals applied on fabric surfaces (if any); 2) fabric interlacing structures; and 3) the surface properties of yarns themselves. Therefore, much information could be neglected if only using SRA and SRW to describe fabric surface properties. The unevenness elements due to these three types of reasons are likely to produced stimulations to receptors with different intensities and frequencies. The Fast Fourier Transform (FFT) was used to obtain such information.

Figure 4 gives a sample of using FFT to extract the fabric surface information. Figure 4(a) is a sample measured curve of the instrument. After the FFT analysis, as shown in Figure 4(b), different sinusoid components are identified and arranged according to their frequencies. Clearly their intensities are different as well. The frequencies of these elements identified are 
based on the measured distance. Since the frequency information that the skin receives is based on time, a transformation was conducted through following formula:

$$
f=v F \quad \text { Equation } 5
$$

where $f$ is frequency based on time, $F$ is frequency based on distance, and $v$ is the scanning speed. When the subjects were evaluating stimuli, their average scanning speed was recorded as $70 \pm 5 \mathrm{~mm} / \mathrm{s}$.

In order to applying the FFT-analyzed fabric surface information into Hypothesis 2 and 3, the maximum investigated frequency is set at $200 \mathrm{~Hz}$, i.e. the minimum repeated distance evaluated is $0.35 \mathrm{~mm}$. The frequency bin applied in this study is $1 \mathrm{~Hz}$. The intensities recorded within the frequency bin are averaged. The thresholds of the PC fibers are extrapolated as a linear function of the vibration frequencies from 10 to $1000 \mathrm{~Hz}$ [24].

Figure 4

Figure caption 4

This characterization method addresses the fabric surface texture with much more details. It agrees with the mechanisms described in Hypothesis 2 and 3, i.e. every uneven element on the fabric surface would stimulate neural signals, although their impacts on the perceived smoothness sensations may be different. Therefore Hypothesis 2 and 3 investigates more on the fabric surfaces, but not only averaging their heights and widths as SRA and SRW do.

Compared to that of Hypothesis 2, hypothesis 3 provides a more direct calculation of the average impulse rates of both the SA1 and PC fibers. While Hypothesis 2 addresses only SA1 fiber, Hypothesis 3 was proposed to calculate both neural fibers. The distinguishing criterion of either SA1 or PC fiber is responsible for smoothness sensation is believed as the width of the unevenness elements [1]. 


\section{Psychological Measurement}

Psychological behavioral measurement was conducted to obtain subjective smoothness sensations of the stimuli. Experiments procedure followed AATCC Evaluation Procedure 5. Subjects washed and dried their hands before the experiments. They then entered the standard experiment room with temperature controlled at $21^{\circ} \mathrm{C} \pm 1{ }^{\circ} \mathrm{C}$ and relative humidity controlled at $65 \% \pm 2 \%$. Totally 226 subjects were recruited. There were 79 males and 147 females. Subjects' average age was 24.5 years old with range from 18 to 60 . No physiological or psychological diseases were reported from subjects. Consents of the experiments were obtained from all participated subjects.

Fabric A was given to subjects as reference fabric for comparison. It was treated with "neutral" smoothness sensation. There were 3 scale points from this midpoint to each side, e.g. rougher or smoother. Subjects were required to compare the other fabrics with the reference fabric. They were asked to touch Fabric A with one hand and the other fabrics with the other hand. Subjects' views were blocked during the experiment. There were 10 descriptors read out by examiners and subjects gave responses. Descriptors included coolwarm, itchy-nonitchy, scratchy-nonscratchy, prickle-nonprickle, smooth-rough, stickynonadhesive, stiff-pliable, thin-thick, soft-hard, and fullness-nonfullness.

Smoothness sensations of them were recorded through factor analysis (FA) on the subjective evaluation results. The results show that first dimensions, named smoothness, could explain $39.4 \%$ of total variances from the 10 descriptors. It includes scratchy-nonsctratchy, pricklenonprickle, itchy-nonitchy, and rouch-smooth. A larger smoothness value means a smoother feeling. 


\section{Results of the Neural Responses}

As shown in Table 2Table, it can be confirmed that there are significant differences between the fabrics examined in terms of all the calculated neural responses. Hypotheses 1 and 3 provide two different methods to calculate SA1 fiber responses. Therefore, a correlation analysis was carried out, as the results shown in Figure 5. It indicates that there is no significant relation. The Spearman's correlation analysis showed that no monotonic relations could be found between them ( $>>0.1)$. Although both methods are similar in principle in that the unevenness of the height and width would affect the response of SA1 fibers, different calculation means have led to different outcomes.

Table 2

Figure 5

Figure caption 5

Meanwhile, Hypotheses 2 and 3 also demonstrate two distinct approaches to characterizing the responses from the PC fibers. As shown in Figure 6, the relation between these two calculated neural responses seems to fit together in a logarithmic function. The Spearman's coefficient is as high as 0.838 . These two methods both examine the unevenness of elements on the fabric surface through sinusoid components after using FFT. This suggests that different degrees of the details examined may be the reason for the previously obtained uncorrelated relation between indexes $I$ and $P_{1}$.

Since Hypothesis 3 addresses both SA1 and PC fibers, their results are verified through a scatter plot as shown in Figure 7. A strong Spearman's correlation (0.983) is observed. In spite of the dissimilar coefficient $k_{i}$ and threshold $T_{i}$, these two fibers demonstrate linear relations with each other. It can be concluded that under this calculation hypothesis of the 
impulse rate of a population of SA1 and PC fibers, the responses of these two types of receptors to stimulation such as those from fabric would be highly correlated.

Figure 6

Figure caption 6

Figure 7

Figure caption 7

Table 3 shows the correlation results between the calculated neural responses and the physical properties of stimuli. The neural responses calculated based on Hypothesis 3 show a significant correlation to both the SRA and SRW physical indexes. The neural responses calculated based on Hypothesis 2 does not show any correlation to the physical indexes while the impulse rate of SA1 fibers based on Hypothesis 1 only shows correlation to SRW.

Table 3

To further examine the relationship between the physical and the neurophysiological indexes, the sinusoid components after using FFT were compared with the SRA and SRW indexes. The highest intensities of the extracted sinusoid components were plotted against the SRA. The frequencies of these components were also verified with the SRW. The results are shown in Table 4. It can be observed that the highest intensity of the extracted sinusoid components shows a high correlation to the SRA. However, the frequency of the components shows no correlation to SRW.

Table 4 
Since the basic concept of textile fabrication is to repeat the interlacing of two groups of yarns, there are two components that may constitute the surface geometric roughness. They include the unevenness of the yarn surface and fabrication structure. According to the definition of the SRA and SRW, these two indexes reflect roughness due to structure since their types of unevenness are usually much greater. Meanwhile, the FFT algorithm classifies various sinusoid components. The components with the highest intensity are therefore considered to be unevenness caused by the fabric structure. A strong correlation found confirms this idea. Nevertheless, the frequency of the component with the highest intensity shows no monotonic relation to the SRW index. This finding implies that despite the strong correlation of the SRA, this pattern recognition method is limited when it comes to characterizing the frequency of unevenness.

In this study, the unevenness of fabrics was measured up to a frequency of $200 \mathrm{~Hz}$. In order to ascertain whether this pre-designed criterion is valid, an analysis was conducted to evaluate the intensity of the components with a frequency greater than $200 \mathrm{~Hz}$. On average the stimuli measured, the intensity of the components at $200 \mathrm{~Hz}$ is only $6 \pm 6 \%$ of the highest intensity. This means that only vibrations with small amplitude could be recorded after 200 Hz. Unevenness at $200 \mathrm{~Hz}$ means that the investigated smallest width of surface unevenness was $0.35 \mathrm{~mm}$.

\section{Discussion}

Spearman's correlation between the calculated neural responses and psychologically obtained smoothness are summarized in Table 5Table. I shows no significant correlation to the smoothness perception. This implies that neural responses to a simple sinusoid model of the surface unevenness are not directly linked to psychological perception. $W$ (identified based 
Hypothesis 2), $P 1$ and $P 2$ (based on hypothesis 3) demonstrate significant correlation to smoothness perception. However, the relation with the highest coefficients of correlation is $W$, which is identified based on Hypothesis 2 of the texture coding mechanism.

Table 5

The scatterplots are shown in Figure 8. It is observed that the relation between $W$ and the smoothness sensation could be better fitted with logarithmic function. This finding is in agreement with previous reports that have used a similar neurophysiological coding algorithm [22]. Based on these observations, a prediction model of smoothness sensation was built. $W$ was chosen as the predictor in the prediction model. Regression result shows that the model has adjusted $\mathrm{R}^{2}$ of 0.615 with $\mathrm{F}$ value of 31.331 and $\mathrm{p}$ value of 0.000 .

Figure 8

Figure caption 8

\section{Model Validation}

For the purpose of the validation of the neuropsychological prediction models, another group of fabrics were evaluated. There were 32 fabrics measured. Physical measurement was conducted by FTT as well while the subjects for psychological measurement included 23 subjects who had also participated previously.

Figure 9 shows the model validation with regard to smoothness. Pearson's correlation coefficients and $\mathrm{p}$ values are displayed at the right bottom of the figure. The Pearson's coefficient of the neuropsychological model is 0.808 with $\mathrm{P}$ values of 0.000 . Result indicates 
that the neuropsychological model can mostly predict the subjective smoothness sensation for this new set of fabrics.

Figure 9

Figure caption 9

\section{Neuropsychological Mechanism}

Figure 10 shows the neuropsychological mechanism of smoothness perception. It can be concluded that smoothness is solely affected by the surface properties of the fabric. As discussed earlier, the surface properties of fabric should be measured by examining its geometric profile. Since our daily contacted surfaces are usually physically heterogeneous, there are many factors that could affect the stimuli's geometric profiles and causing surface unevenness in different scales. Therefore, the FFT analysis should be used to classify the surface unevenness into different sinusoid components with various intensities and frequencies. The natural of the afferent fibers implies that different intensities and frequencies will lead to different firing responses of the fibers. Findings in this report prefer one of the neurophysiological coding mechanisms, which indicating that the weighted summation of these elements could be responsible for the response power of PC fibers. The weights of these elements vary according to their frequency.

Figure 10

Figure caption 10

\section{Conclusion}

This report summaries three different extracted neurophysiological mechanisms of surface texture smoothness perception. They were modified according to the unique feature of fabrics and the calculated biomimetic neural responses were examined through comparison with 
psychological ratings. Findings from the experiments suggest that the simple linear coding of SA1 spatial variances only have limited correlation to subjective ratings. This hypothesis applies a simplified recognition method of fabric surface unevenness, which considers them as repeating of a single sinusoid unit. Whilst biomimetic neural responses calculated based on both second and third hypotheses demonstrate significant correlation to subjective ratings. These two hypotheses use a similar characterization method of the fabric surface unevenness, which is distinguishing them into different intensity with different frequency through FFT algorithm. In addition, results show that there is closed correlation between biomimetic neural responses of SA1 fibers and PC fibers according to hypothesis three. Although their firing patterns are different, it seems their responses to heterogeneous surfaces are similar. Therefore, this report suggests that it is important to characterize different components of heterogeneous surface roughness and accumulate their impacts based on the sensitivities to different stimulus frequencies of neural fiber. It is expected that this study could be further expanded to investigate other types of touch perceptions and more importantly, dynamic perception of touch comfort through similar neuropsychological methods. 


\section{Reference}

1. A. I. Weber, H. P. Saal, J. D. Lieber, J. W. Cheng, L. R. Manfredi, J. F. Dammann, and S. J. Bensmaia, P Natl Acad Sci USA, 110, 17107 (2013).

2. I. L. Ciesielska-Wrobel and L. Van Langenhove, Text. Res. J., 82, 1457 (2012).

3. X. Liao, J. Hu, Y. Li, Q. Li, and X. Wu, Journal of Fiber Bioengineering \& Informatics, 4, 105 (2011).

4. $\quad$ R. S. Johansson and A. B. Vallbo, Trends in Neurosciences, 6, 27 (1983).

5. $\quad$ S. S. Stevens and J. R. Harris, J Exp Psychol, 64, 489 (1962).

6. $\quad$ S. J. Lederman, Percept Psychophys, 16, 385 (1974).

7. S. J. Lederman, Canadian journal of psychology, 37, 498 (1983).

8. K. Sathian, A. W. Goodwin, K. T. John, and I. Dariansmith, J Neurosci, 9, 1273 (1989).

9. D. T. Blake, S. S. Hsiao, and K. O. Johnson, J Neurosci, 17, 7480 (1997).

10. C. E. Connor, S. S. Hsiao, J. R. Phillips, and K. O. Johnson, J Neurosci, 10, 3823 (1990).

11. C. E. Connor and K. O. Johnson, J Neurosci, 12, 3414 (1992).

12. T. Yoshioka, B. Gibb, A. K. Dorsch, S. S. Hsiao, and K. O. Johnson, J Neurosci, 21, 6905 (2001).

13. X. Liao, Y. Li, J. Y. Hu, X. X. Wu, and Q. H. Li, Fiber. Polym., 15, 1548 (2014).

14. J. Phillips, R. Johansson, and K. Johnson, Experimental Brain Research, 81, 589 (1990).

15. J. J. DiCarlo and K. O. Johnson, J Neurosci, 20, 495 (2000).

16. D. T. Blake, K. O. Johnson, and S. S. Hsiao, Journal of Neurophysiology, 78, 2503 (1997).

17. M. Hollins and S. R. Risner, Percept Psychophys, 62, 695 (2000).

18. M. Hollins and A. Sigurdsson, Pain, 75, 59 (1998).

19. M. Hollins, S. J. Bensmaia, and E. A. Roy, Behav Brain Res, 135, 51 (2002).

20. S. J. Bensmaia and M. Hollins, Somatosens Mot Res, 20, 33 (2003).

21. J. C. Makous, R. M. Friedman, and C. J. Vierck, J Neurosci, 15, 2808 (1995).

22. S. Bensmaia and M. Hollins, Percept Psychophys, 67, 842 (2005).

23. S. J. Bolanowski, G. A. Gescheider, R. T. Verrillo, and C. M. Checkosky, J Acoust Soc Am, 84, 1680 (1988).

24. M. A. Muniak, S. Ray, S. S. Hsiao, J. F. Dammann, and S. J. Bensmaia, J Neurosci, 27, 11687 (2007). 


\section{Table}

Table 1 Fabric Samples List

\begin{tabular}{|c|c|c|c|c|c|}
\hline \multirow[t]{2}{*}{$\mathbf{S} / \mathbf{N}$} & \multirow[t]{2}{*}{ Fabric Description } & \multicolumn{2}{|c|}{$\begin{array}{c}\text { Weight } \\
\left(\mathrm{g} / \mathrm{m}^{2}\right)\end{array}$} & \multicolumn{2}{|c|}{$\begin{array}{c}\text { Thickness } \\
\text { (mm) }\end{array}$} \\
\hline & & Mean & S.D. & Mean & S.D \\
\hline 1 & $100 \%$ Cotton Plain Woven & 127.7 & 0.8 & 0.39 & 0.01 \\
\hline 2 & 100\% Linen Plain Woven & 203.1 & 2.0 & 0.59 & 0.02 \\
\hline 3 & $100 \%$ Cotton Non-Woven & 33.8 & 1.9 & 0.37 & 0.03 \\
\hline 4 & 100\% Cotton Twill Woven & 265.5 & 1.3 & 0.70 & 0.05 \\
\hline 5 & $100 \%$ Polyester Chiffon Knitted & 78.3 & 0.9 & 0.22 & 0.00 \\
\hline 6 & 100\% Wool Satin Woven & 252.5 & 2.1 & 0.34 & 0.00 \\
\hline 7 & $60 \%$ Polytester/ $40 \%$ Wool Flannel Woven & 340.8 & 2.4 & 1.29 & 0.01 \\
\hline 8 & $94 \%$ Cotton $6 \%$ Elasthan 3 X3 RIB & 123.5 & 3.5 & 1.30 & 0.02 \\
\hline 9 & 90\% Polyester/10\% Elasthan Single Jersey & 178.1 & 2.9 & 0.26 & 0.00 \\
\hline 10 & $75 \%$ Viscose $15 \%$ Nylon $10 \%$ Elasthan Single Jersey & 157.7 & 2.2 & 0.52 & 0.01 \\
\hline 11 & $56 \%$ Cupro 24\% Nylon 20\% Elasthan Single Jersey & 137.7 & 1.0 & 0.34 & 0.04 \\
\hline 12 & $96 \%$ Tencel 4\% Elasthan Jacquard & 144.7 & 2.1 & 1.15 & 0.04 \\
\hline 13 & 45\% Polyester $41 \%$ Nylon $14 \%$ Elasthan Jacquard & 112.5 & 1.1 & 0.88 & 0.02 \\
\hline 14 & 95\% Nylon 5\% Elasthan Single Jersey & 154.9 & 1.2 & 0.58 & 0.00 \\
\hline 15 & 100\% Silk Jacquard Knitted & 142.3 & 0.5 & 0.59 & 0.02 \\
\hline 16 & 100\% Silk Jacquard Knitted & 139.8 & 1.0 & 0.56 & 0.01 \\
\hline 17 & 100\% Silk Jacquard Knitted & 122.3 & 0.5 & 0.36 & 0.00 \\
\hline 18 & 100\% Silk Jacquard Knitted & 117.4 & 0.9 & 0.48 & 0.00 \\
\hline 19 & 100\% Silk Jacquard Knitted & 169.5 & 0.6 & 0.63 & 0.01 \\
\hline
\end{tabular}


Table 2 Summary of ANOVA Test on Calculated Neural Responses

Table 3 Correlation Results between the Biomimetic Neural Responses and the Physical Properties

\begin{tabular}{llrrrr}
\hline Index & Statistic & $\boldsymbol{I}($ SA1 $)$ & $W($ PC) & $\boldsymbol{P}_{\mathbf{1}}(\mathbf{S A 1})$ & $\boldsymbol{P}_{2}($ PC) \\
\hline SRA & Coefficient & -- & -- & .481 & .573 \\
& p Value & -- & -- & .032 & .008 \\
\multirow{2}{*}{ SRW } & Coefficient & -.522 & -- & .448 & .489 \\
& p Value & .018 & -- & .048 & .029
\end{tabular}

Table 4 Correlation Results between Measured Physical Properties of the Stimuli and Frequency and Intensity of Sinusoid Elements with Highest Intensity

\begin{tabular}{llcr}
\hline Physical Index & Statistic & Frequency & Intensity \\
\hline SRA & Coefficient & -- & .940 \\
& p Value & -- & .000 \\
\multirow{2}{*}{ SRW } & Coefficient & -- & .758 \\
& p Value & -- & .000 \\
& & & \\
\hline
\end{tabular}


Table 5 Correlation Results between Biomimetic Neural Responses and Psychological

obtained Smoothness Sensations

\begin{tabular}{llr}
\hline Neural & Statistic & Smoothness \\
Responses & & -- \\
\hline $\mathrm{I}$ & Coefficient & -- \\
& $\mathrm{p}$ Value & -0.738 \\
$\mathrm{~W}$ & Coefficient & 0.000 \\
& $\mathrm{p}$ Value & -0.684 \\
$\mathrm{P}_{1}$ & Coefficient & 0.001 \\
& $\mathrm{p}$ Value & -0.620 \\
& Coefficient & \\
$\mathrm{P}_{2}$ & $\mathrm{p}$ Value & 0.004 \\
& & \\
\hline
\end{tabular}




\section{Figure captions}

Figure 1 Perception Process of Touch Smoothness

Figure 2 Sample Curve of FTT Roughness Measurement

Figure 3 Sinusoid Characterization of Surface Unevenness

Figure 4 Fast Fourier Transform Algorithm on Fabric Surface Profile: (a) original FTT measured surface profile curve is made of discrete points along measured distance (x axis); (b) Spectrum curve after FFT with $\mathrm{x}$ axis as frequency and y axis as intensity; (c) Intensities in frequency bin of $1 \mathrm{~Hz}$ are averaged and plotted as bar chart.

Figure 5 Relation Scatterplot of Coded SA1 Fiber Responses I and P1

Figure 6 Scatterplot of Coded PC Fiber Responses W and P2

Figure 7 Relation Scatterplot of Coded SA1 (P1) and PC (P2) Fiber Population Impulses Rate Figure 8 Scatterplots of Biomimetic Neural Responses and the Smoothness Sensation Figure 9 Scatterplot of the predicted smoothness sensation values and the psychologically obtained values

Figure 10 Neuropsychological Mechanism of Perceiving Smoothness Sensation 


\section{Figure}

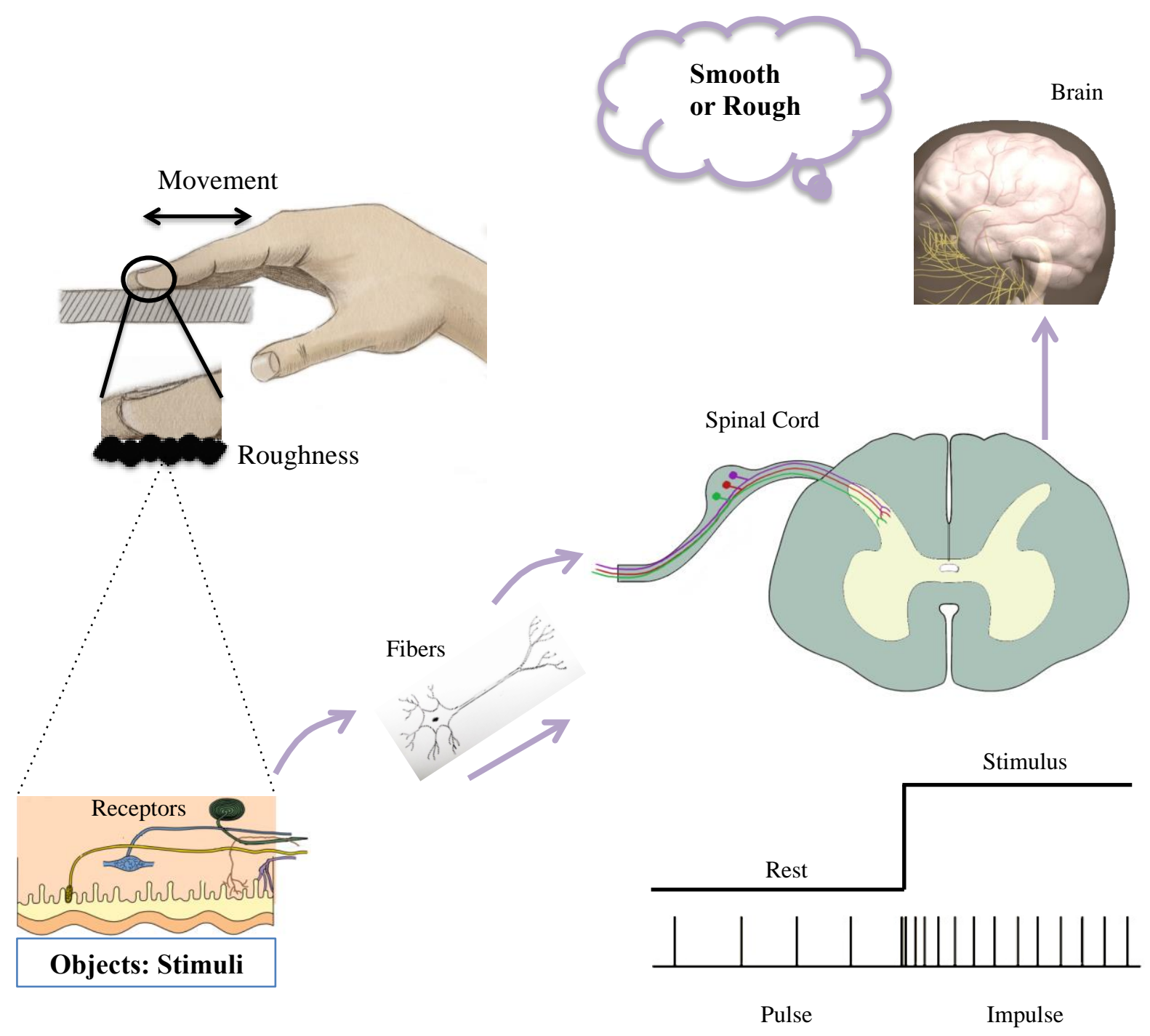

Figure 1

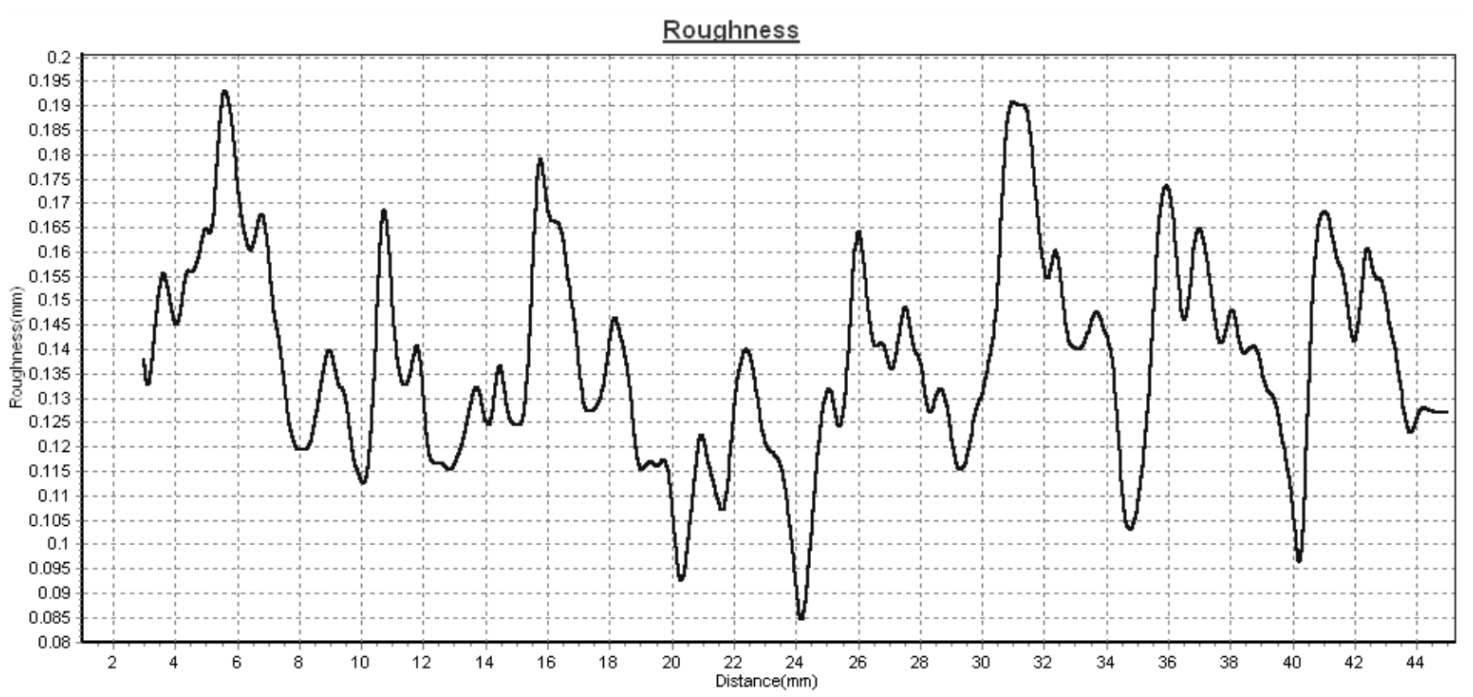


Figure 2

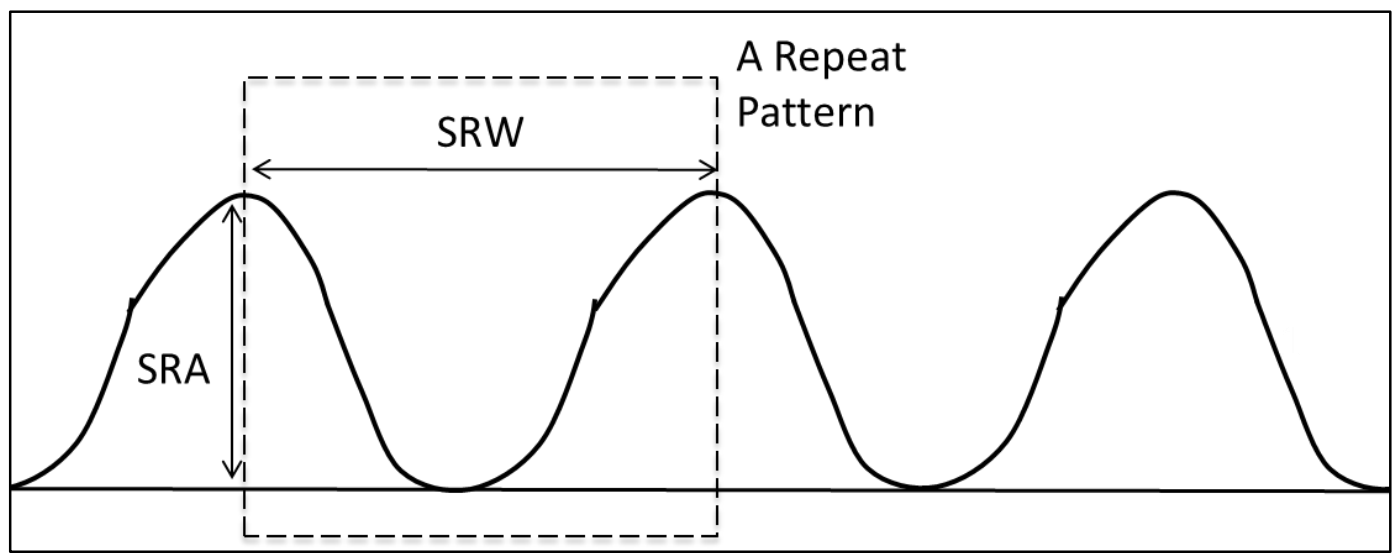

Figure 3

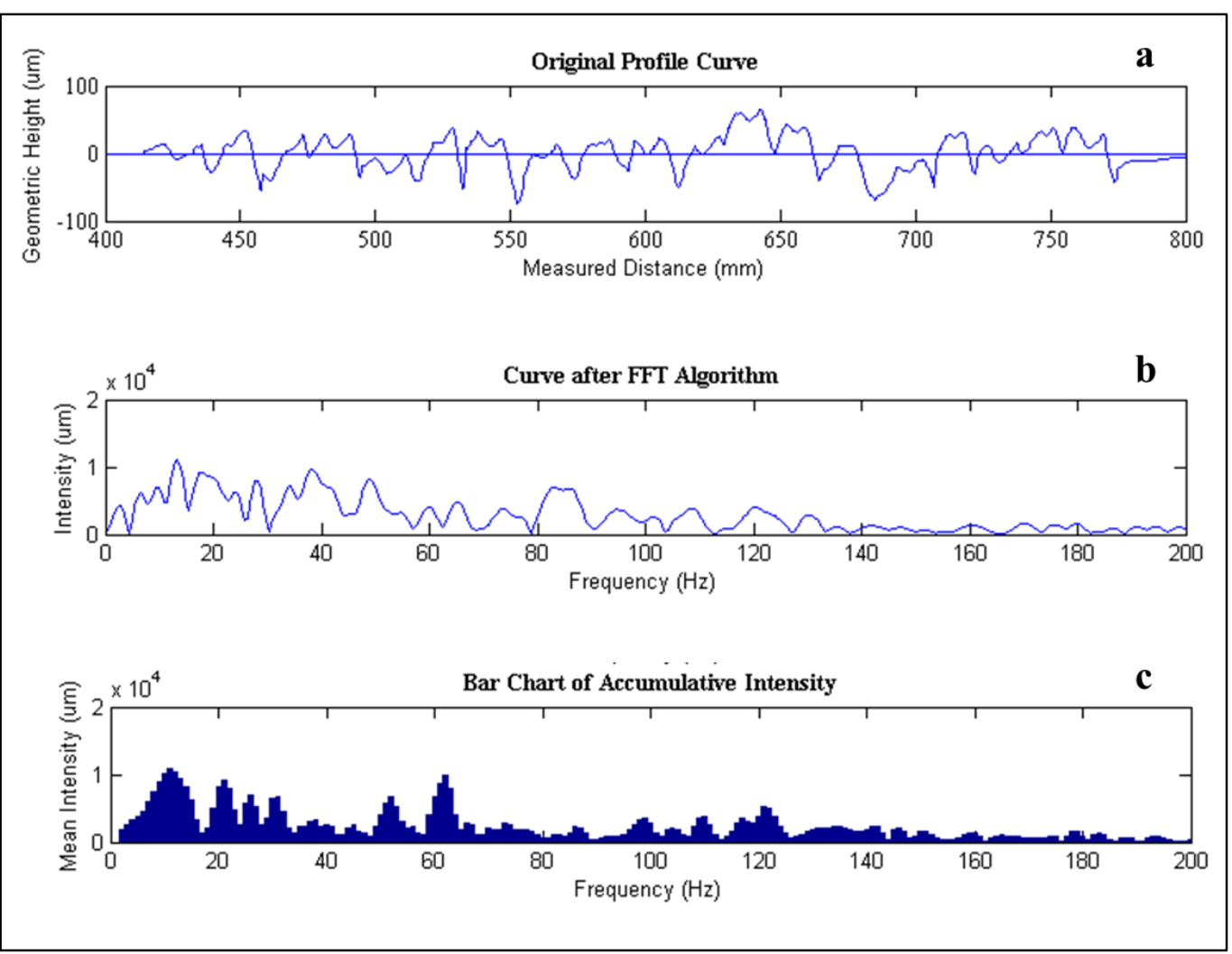

Figure 4 


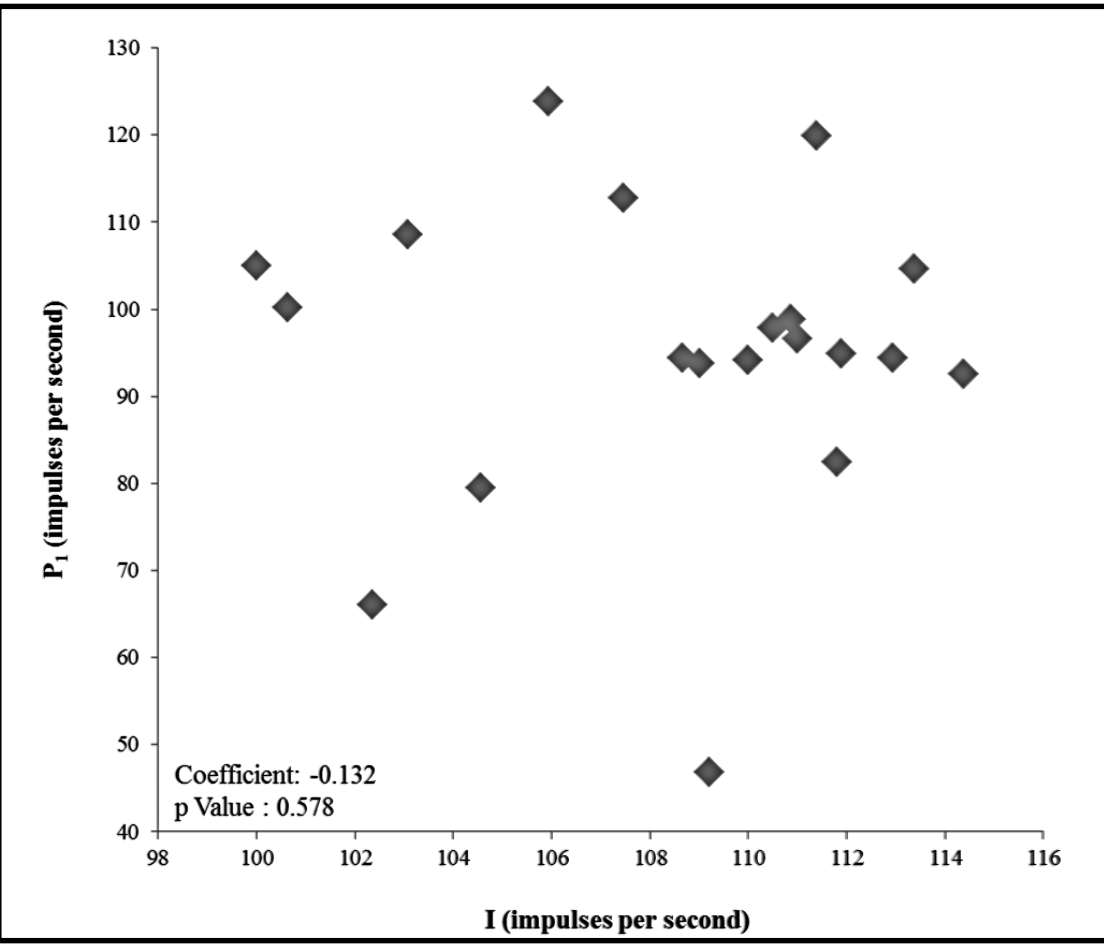

Figure 5

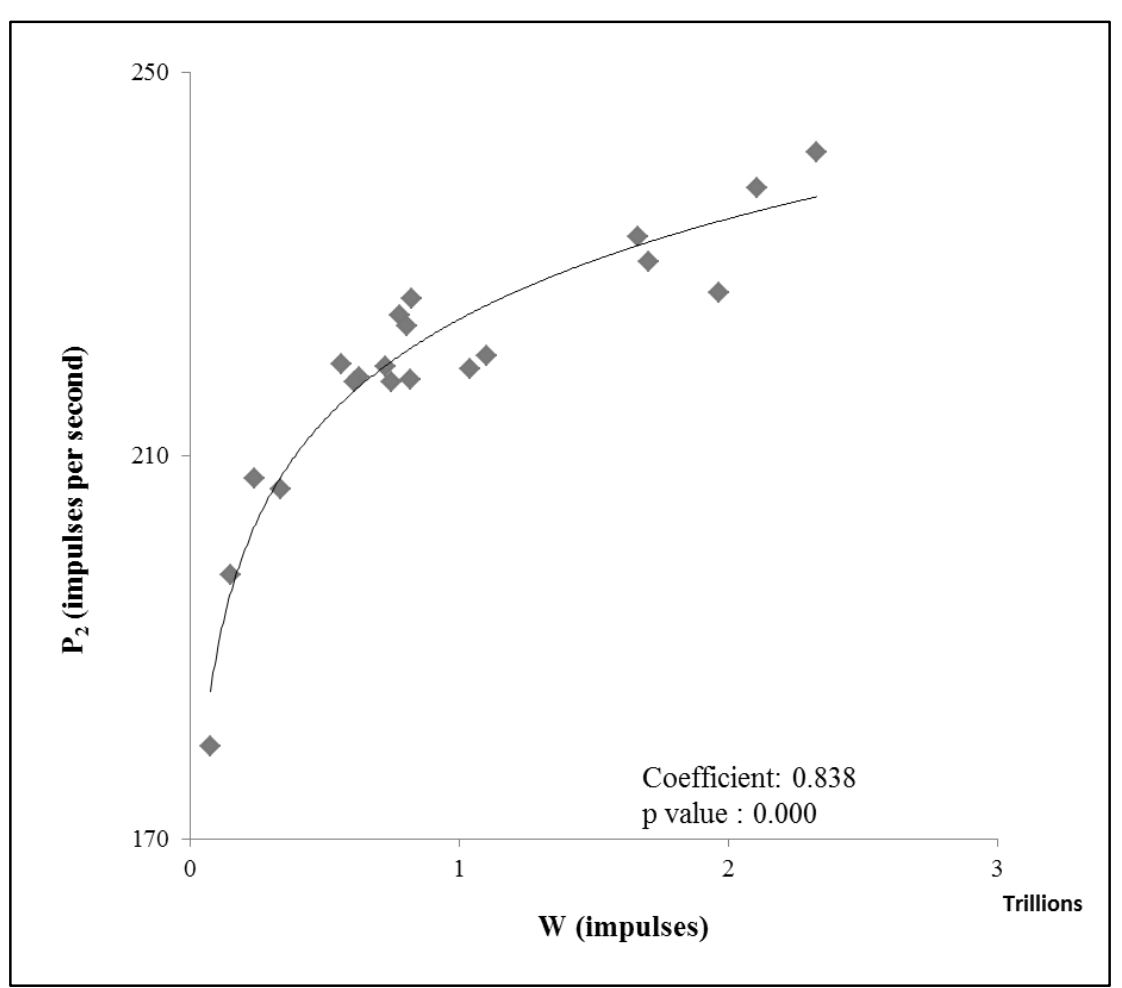

Figure 6 


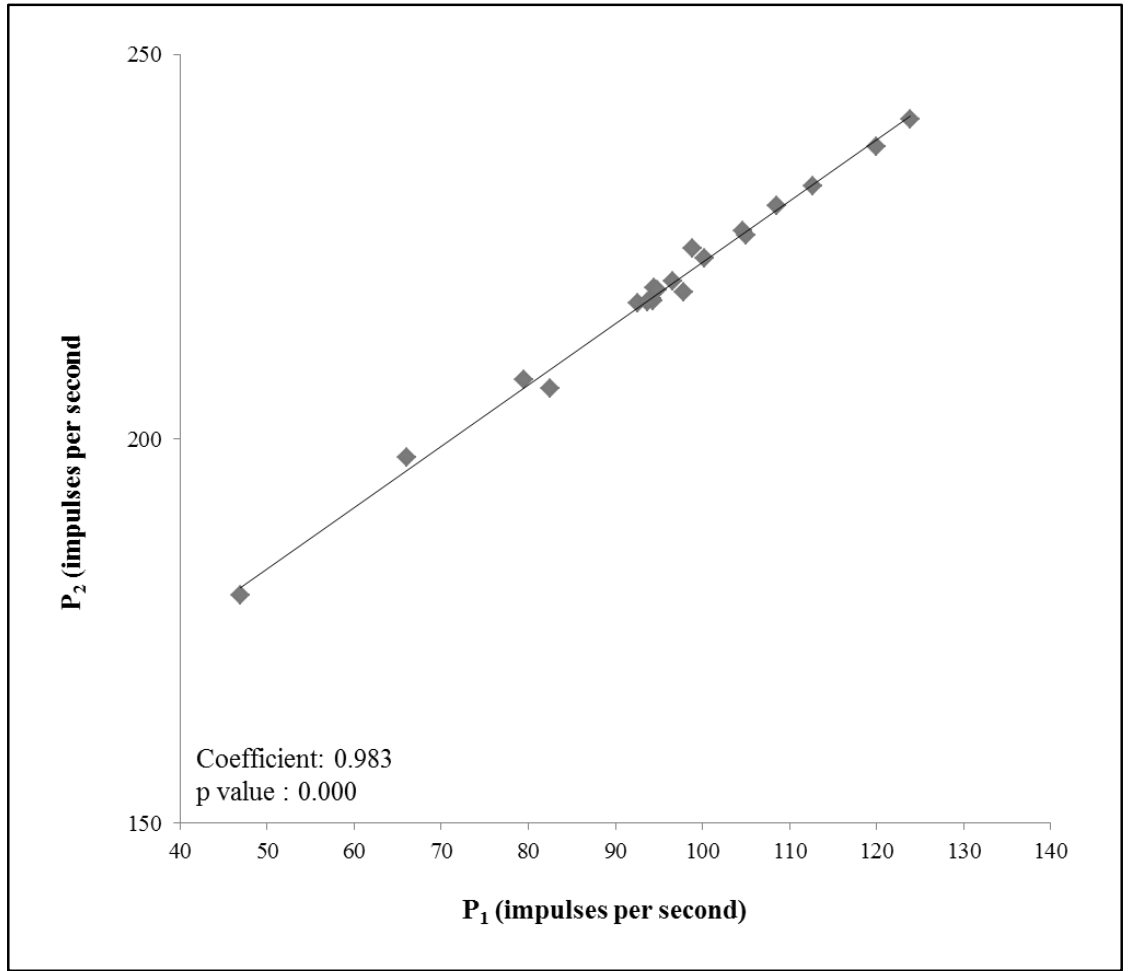

Figure 7

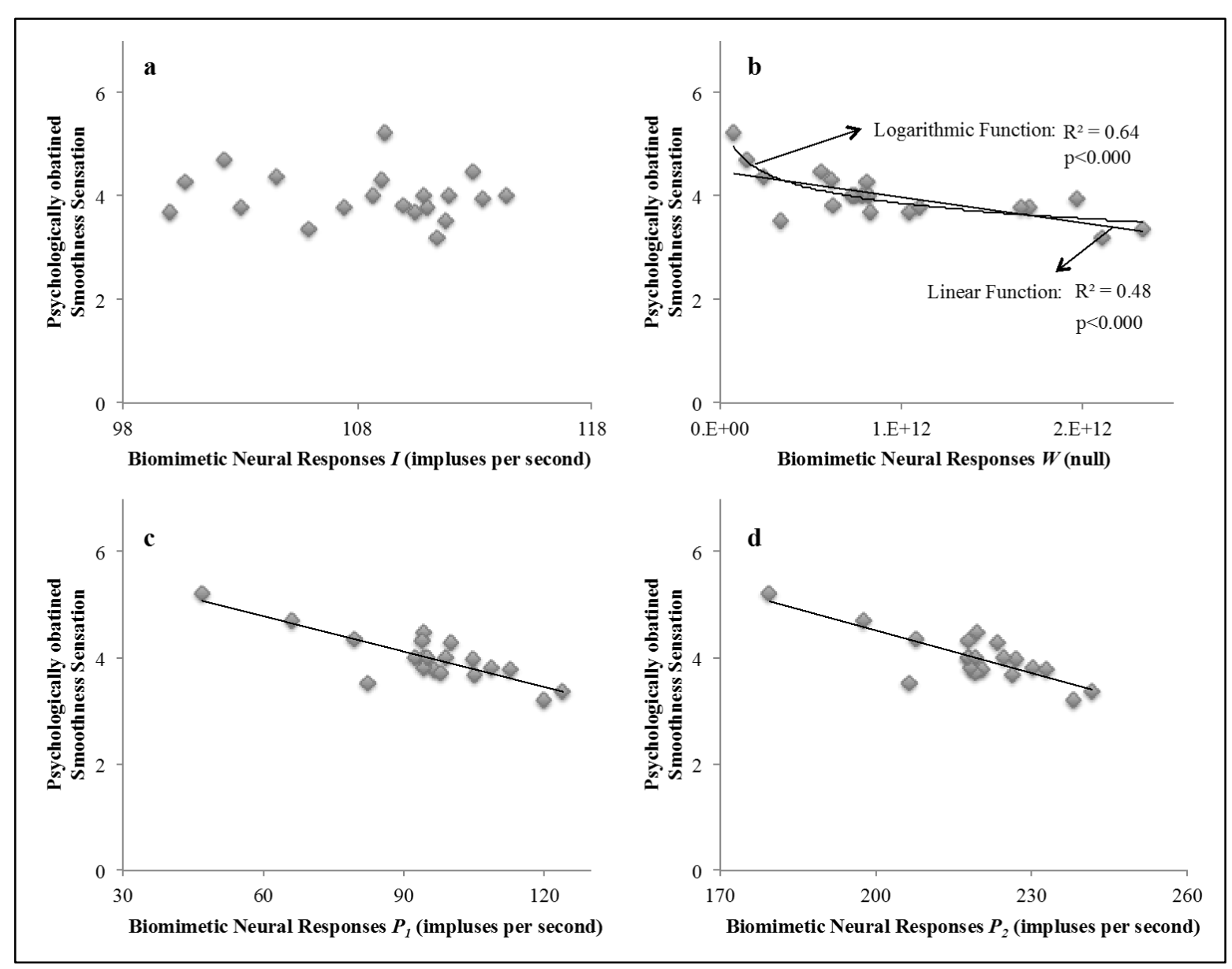

Figure 8 


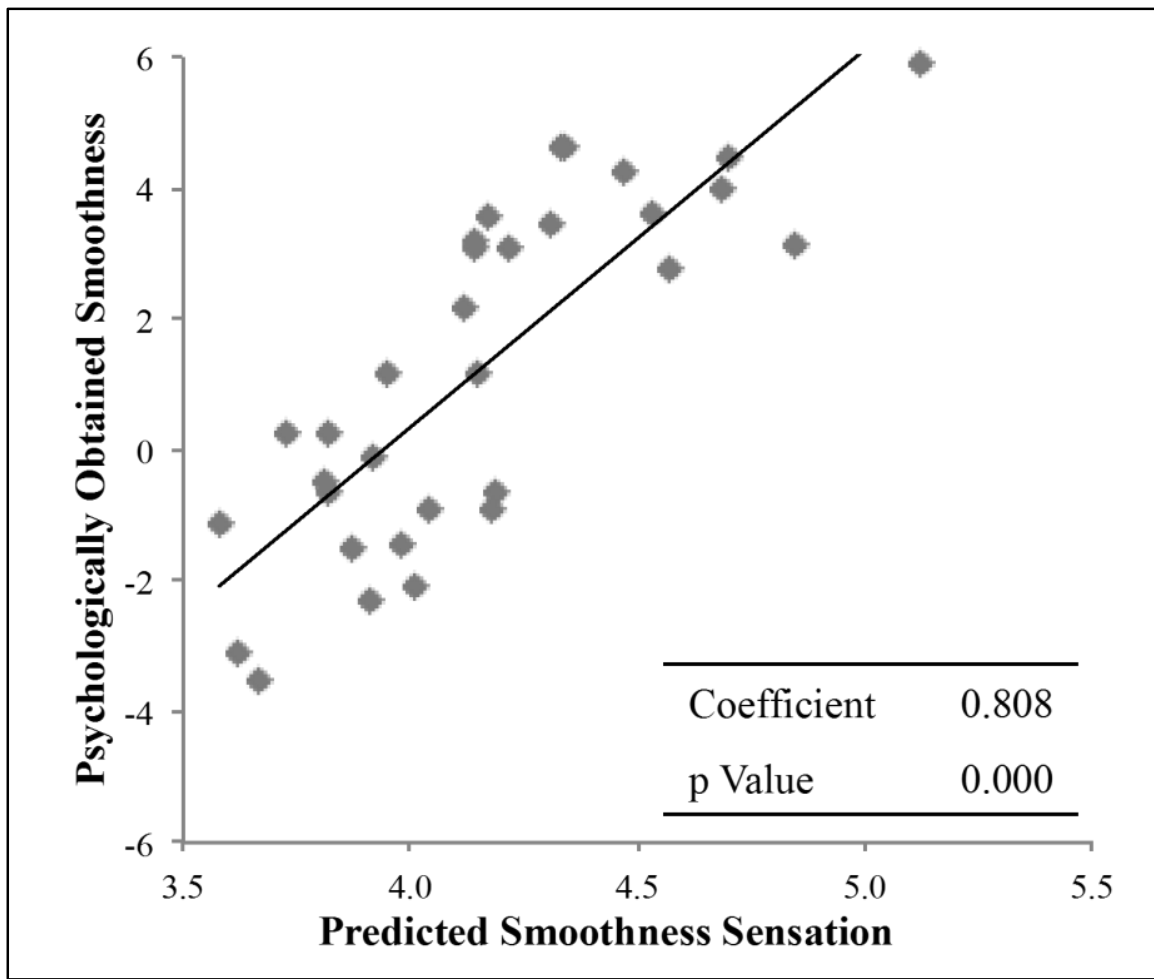

Figure 9

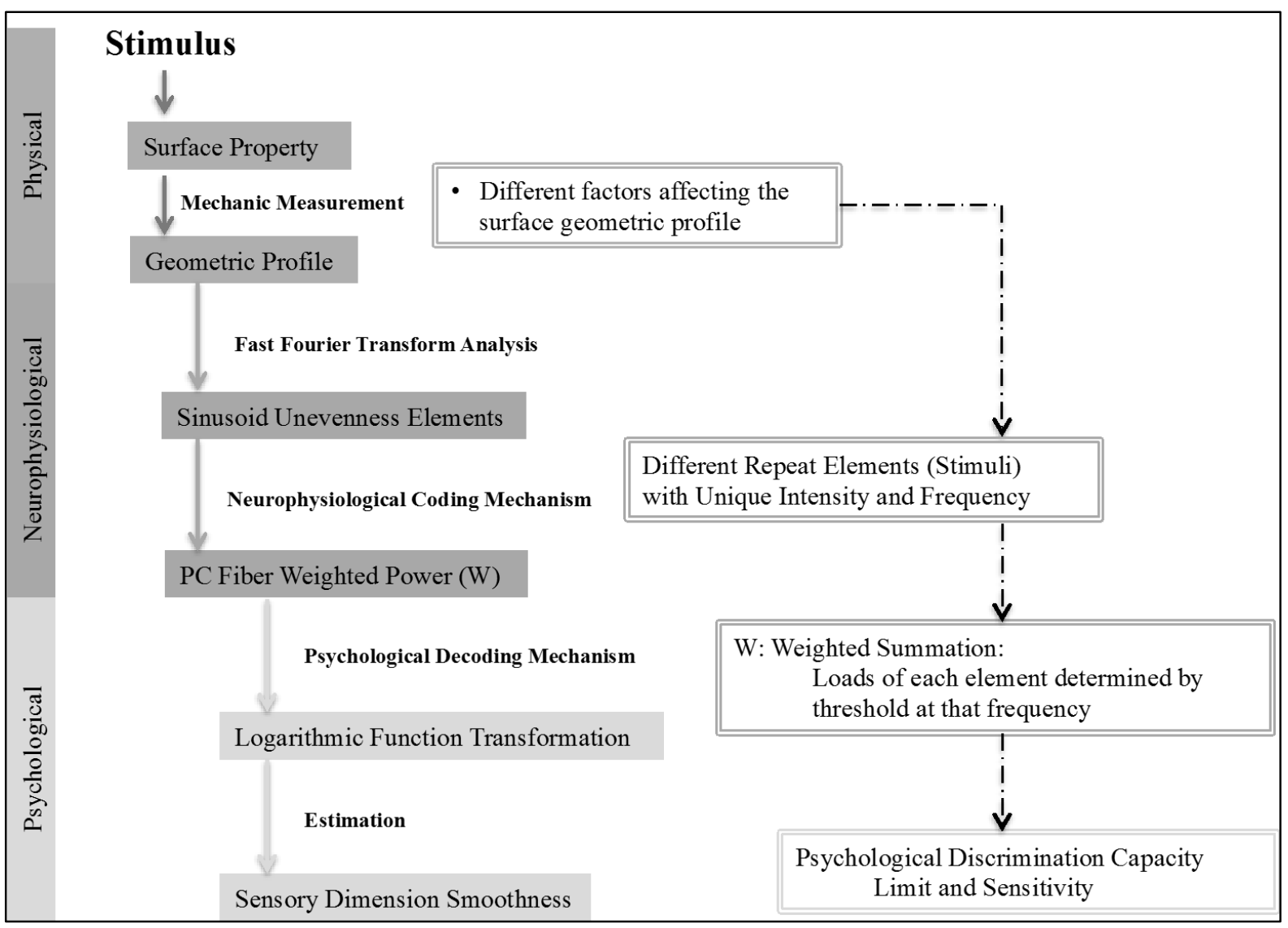

Figure 10 